\title{
Clinical Efficacy of Tildrakizumab in Patients With Chronic Plaque Psoriasis Over 2 Years of Treatment: Results From Long-term Extensions to 2 Phase 3 Clinical Studies
}

Kim Papp, ${ }^{1}$ Kristian Reich, ${ }^{2}$ Andrew Blauvelt, ${ }^{3}$ Diamant Thaçi, ${ }^{4}$ Rodney Sinclair, ${ }^{5}$ Stephen K Tyring, ${ }^{6}$ Nicole Cichanowitz, ${ }^{7}$ Stuart Green, ${ }^{7}$ Qing Li, ${ }^{7}$ Carmen La Rosa ${ }^{7}$

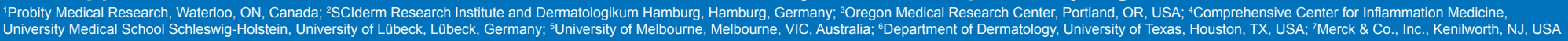

\section{BACKGROUND}

- Tildrakizumab is a aigh-aftinity humanized, anti-l-23p19 (1)

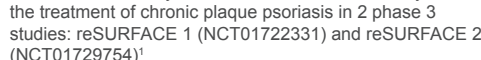
(NCT01729754)

- Both of hhese studies have an optional long-term extension,
each with an additional treatment period of up to 192 weeks

\section{OBJECTIVE}

Preliminary evaluation of maintenance of response data in
patients who were responders to tildrakizumab upon enterin the extension periods and who maintained response a year
into the extensions (a total of at least 2 years of treatment

\section{METHODS}

Base Studies

The reSURFACE base studies are 3-part, double-blinded, randomized, placebo-controlled studies in patients with

Inclusion criteria included age $\geq 18$ years, body surface area score $\geq 3$, and Psoriasis Area and Severity Index (PASI) $\geq 1$ In the base studies, tildrakizumab 200 and $100 \mathrm{mg}$ were
evaluated for 64 weeks (reSURFACE 1) and 52 weeks evaluated for 64
(reSURFACE 2)

In Part 1 of the studies (Weeks 1-12), patients were
randomized to subcutaneous tildrakizumab $200 \mathrm{mg}$. tildrakizumab $100 \mathrm{mg}$, or placebo, and treatment was was an additional treatment arm of etanercept $50 \mathrm{mg}$ administered twice weekly

In Part 2 (Weeks 12-28), patients previously receiving $100 \mathrm{mg}$ and received trzeed to tildrakizumab $200 \mathrm{mg}$ or In reSURFACE 2 , the dose in the etanercept arm was $50 \mathrm{mg}$ once weekly

Extension Studies

Patients completed the base studies and chose to

Patients achieved $250 \%$ improvement in PASI (PASI 50

In reSURFACE 1 only, patie active dose of tildrakizumab within 12 weeks of the end of

Patients received the same dose of tildrakizumab (200 or of the base studies; administration was open label after

Efficacy objective for the extension period:
- Evaluation of the maintenance of efficacy endpoints

(ie, proportion of PASI $50,75,90$, and 100 responders
1 year into the extension among PASI 50, 75, 90, and 100 .
- Prespecified to be based on observed data, with no
statistical analyses planned for comparison between dos

The primary efficacy population was the full analysis set, defined as patients with at least 1 dose of extensio treatment based on assigned treatment

Safety objective for the extension period:
- Evaluation of adverse events (AEs) for up to 5 years; prespecified AEs of interest were summarized by
putions

treatment over time

Yearly and cumulative (base and extension combined)

\section{RESULTS}

In reSURFACE 1, 772 patients entered the study,
638 patients completed the base period, and 506 patients
entered the extension; in reSURFACE 2, 1090 patients entered the extension; in reSURFACE 2, 1090 patients
entered the study, 756 patients completed the base period, entered the study, 756 patients completed the base pe

Figure 1. Patient Flow

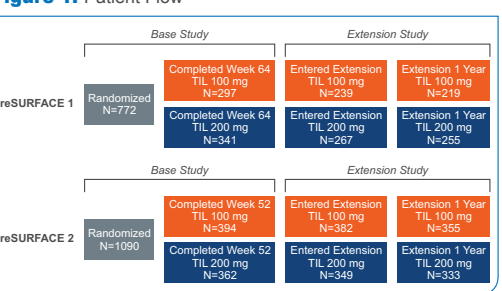

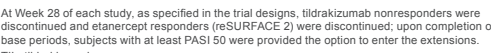

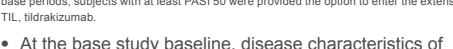

patients who went on to enter the extension were similar
between the 2 studies, and between the tildrakizumab 100 -n between the 2 studies, and be
and $200-\mathrm{mg}$ groups (Table 1 )

The percentage of white patients was lower in
reSURFACE 1 than 2 because reSURFACE 1 included sites in Japan, whereas reSURFACE 2 did not

Efficacy

PASI responses were maintained in most patients from the end of the base period through the extension period: In reSURFACE 1, in patients entering the extension
on tiddrakizumab $200 \mathrm{mg}$. PASI $50 / 75 / 90 / 100$ was maintained by $97 \% / 91 \% / 82 \% / 63 \%$ (out of $255 / 208 / 135 / 70$ patients with data at 1 year); in patients on tildrakizumab $98 \% / 90 \% / 74 \% / / 53 \%$ (out of $219 / 195 / 121 / 70$ patients with data at 1 year) (Figure 2A)

In reSURFACE 2, in patients entering the extension on tildrakizumab $200 \mathrm{mg}$, PASI 50/75/90/100 was patients with data 11 rearl) for those on (330/293/191/97 $100 \mathrm{mg}$. PASI $50 / 75 / 900 / 100$ was maintained by
$99 \% / 92 \% / 84 \% / 66 \%$ (out of $352 / 327 / 249 / 125$ patients with $99 \% / 92 \% / 84 \% / 66 \%$ (out of
data at 1 year) (Figure 2 B)

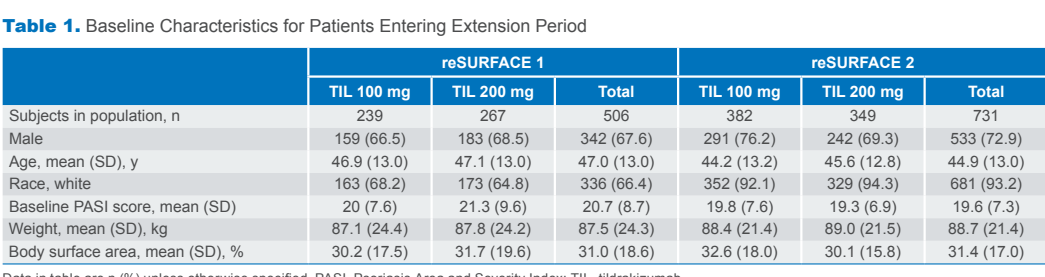
atati in table are $n(\%)$ unless otherwise specified. PASI, Psoriasis Area and Severity Index; TL, tildrakizumab

- After 2 years of treatment with tildrakizumab, including the base and extension periods, $84 \%$ and $88 \%$ of patients who received groups, $81 \%$ and $84 \%$ of patients achieved PASI 75 in reSURFACE 1 and reSURFACE 2, respectively (Figure 3 )

Figure 2. Maintenance of PASI 75 Response From End of Figure 3. Overall Efficacy After 2 Years of Treatment and (B) reSURFACE 2
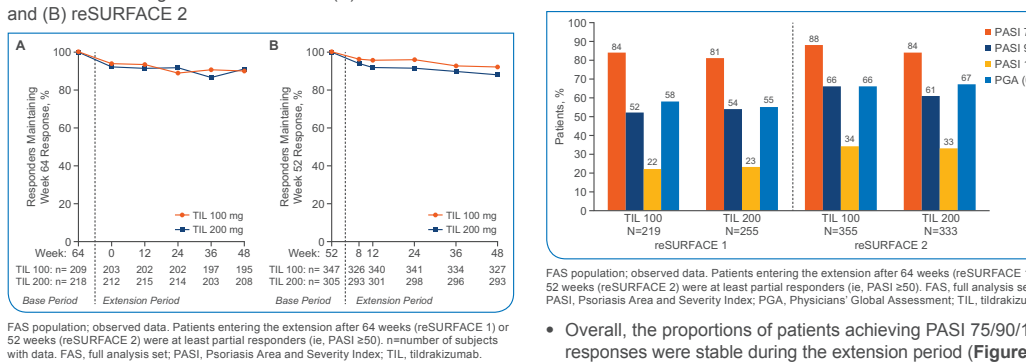

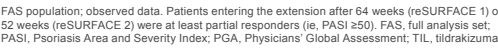
- Overall, the proportions of patients achieving PASI 75/90/100
responses were stable during the extension period (Figure 4 ) Figure 4. Overall Efficacy From End of Base Period Through Extension Periods (A) reSURFACE 1 and (B) reSURFACE 2

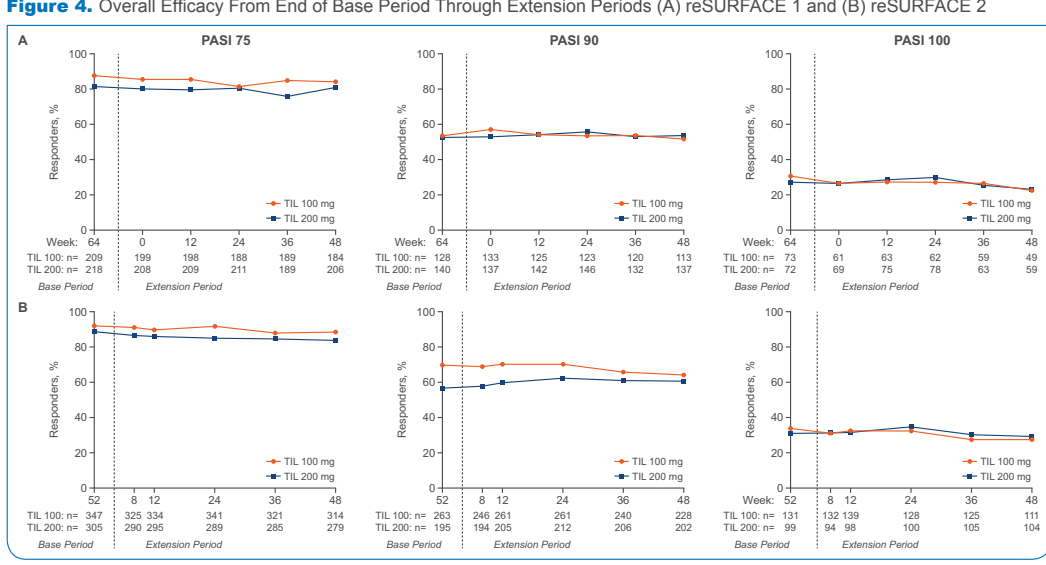

Safety

- After 2 years of treatment with tildrakizumab, the cumulative
number of patients with prespecified $A$ Es was low in both the 200-mg and 100-mg groups in both studies (Table 2) Table 2. Two-Year Cumulative Number (Rate) of Patients

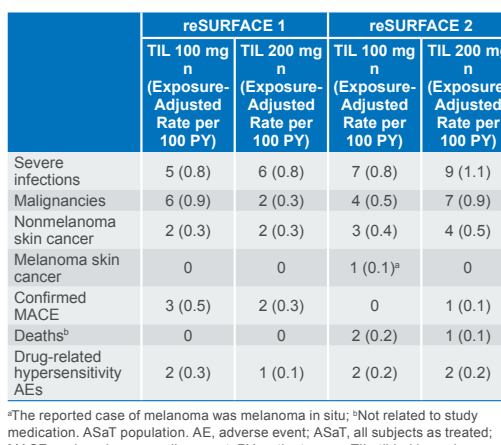
medication. ASaT population. AE, adverse event; ASaT, all subjects as treated

\section{CONCLUSIONS}

- Tildrakizumab $100 \mathrm{mg}$ or $200 \mathrm{mg}$ demonstrated maintenance of efficacy in the treatment of moderate to severe
plaque psoriasis for at least 2 years of treatment

Dver a cumulative 2-year treatment period in patients

200 and $100 \mathrm{mg}$ were well-tolerated with a low rate of AEs of interest

REFERENCES

ACKNOWLEDGMENTS

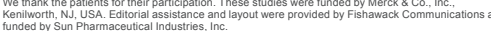

DISCLOSURES



